\title{
Avaliação da prevalência de suicídio em Joinville (SC) no período de 2016 a 2018
}

\author{
Assessment of the prevalence of suicide in Joinville (SC) from 2016 to 2018 \\ Evaluación de la prevalencia del suicidio en Joinville (SC) de 2016 a 2018
}

Aline Plucinski ${ }^{1 *}$, Victória Cenci Guarienti ${ }^{1}$, Tuanne Marques Pereira1, Fabiane Suel de Borba Farikoski $^{2}$, Januária Ramos Pereira Wiese ${ }^{1}$.

\section{RESUMO}

Objetivo: Analisar dados referentes à mortalidade por suicídio, incluindo o estudo sobre o perfil populacional com maior taxa de acometimento na cidade de Joinville, Santa Catarina, Brasil, no período de 2016 a 2018. Métodos: Os dados foram obtidos através das informações contidas nas bases do Núcleo de Prevenção à Violência a partir das notificações do Sistema de informação de Agravos de Notificação (SINAN), foram selecionadas e divididas em idade, sexo, raça, escolaridade, bairro residente, local do suicídio e CID aplicado, as variáveis foram analisadas e detalhadas no decorrer do artigo. Resultados: Os resultados obtidos foram de que o principal perfil populacional acometido pelos óbitos por suicídio foi de homens na faixa etária de 60 anos ou mais, seguido por um aumento significativo na taxa de mortalidade em jovens estudantes. Considerações finais: Constatado então o perfil populacional na taxa de mortalidade, estratégias de prevenção direcionadas podem ser criadas, além de também aprimorar a rede de tratamento e acolhimento destas vítimas.

Palavras-chave: Suicídio, Mortalidade, Prevenção.

\begin{abstract}
Objective: Analyze data on mortality by suicide, including a study on the profile of the population with the highest suicidal rate in the city of Joinville, Santa Catarina, Brazil, in the period from 2016 to 2018 . Methods: Data was obtained through information contained in the databases of the Violence Prevention Center, from the notifications of the Sistema de informação de Agravos de Notificação (SINAN). The cases were selected and divided into age, gender, race, education, resident neighborhood, place of suicide and ICD. The variables were analyzed and detailed throughout the article. Results: The results obtained showed that the main population affected by suicide deaths were men aged 60 or higher, followed by a significant increase in the mortality rate in young students. Final considerations: Once the population profile is verified in the mortality rate, targeted prevention strategies can be created, in addition to improving the treatment and reception network for these victims.
\end{abstract}

Key words: Suicide, Mortality, Prevention.

\section{RESUMEN}

Objetivo: Analizar los datos sobre la mortalidad por suicidio, incluyendo el estudio sobre el perfil de la población con mayor tasa de afectación en la ciudad de Joinville, Santa Catarina, Brasil, en el período de 2016 a 2018. Métodos: Los datos se obtuvieron a través de la información contenida las bases del Centro de Prevención de la Violencia a partir de las notificaciones del Sistema de Información de Enfermedades Notificables (SINAN), fueron seleccionadas y divididas por edad, género, raza, educación, barrio de residencia, lugar de suicidio y aplicada la CIE, las variables fueron analizadas y detalladas a lo largo del artículo. Resultados: Los resultados obtenidos fueron que el principal perfil poblacional afectado por muertes por suicidio eran los hombres de 60 años o más, seguido de un aumento significativo em la tasa de mortalidad em los jóvenes estudiantes. Consideraciones finales: Una vez verificado el perfil de la población en la tasa de mortalidad, se pueden crear estrategias de prevención focalizadas, además de mejorar la red de atención y recepción de estas víctimas.

Palabras clave: Suicidio, Mortalidad, Prevención.

1 Universidade da Região de Joinville (UNIVILLE), Joinville - SC. *E-mail: alineplucinski@gmail.com

2 Vigilância Epidemiológica de Joinville, Joinville - SC.

SUBMETIDO EM: 8/2021

PUBLICADO EM: 8/2021 


\section{INTRODUÇÃO}

O suicídio é considerado um sério problema de saúde pública sendo classificado como uma das dez principais causas de óbito na população mundial independente da faixa etária analisada (SOUZA VS, et al., 2011). O comportamento suicida pode resultar ou não no ato do suicídio. Ele é definido como sendo um ato intencional de causar dano a si mesmo, "dar fim a própria vida voluntariamente", por ser um fenômeno paradoxal, desafia várias áreas do conhecimento, religião e senso comum (DAOLIO ER e SILVA JV, 2009). É um fenômeno multicausal, que envolve inúmeras condições como fatores ambientais, psicológicos, culturais, biológicos e políticos na existência da ideação suicida (SCHOLOSSER A, et al., 2014).

De acordo com a Organização Mundial da Saúde (OMS) são contabilizados 703.000 óbitos devido ao suicídio todos os anos sendo este número superior aos óbitos ocasionados por doenças como malária, HIV / AIDS e câncer de mama além de guerras e homicídios. Mais de uma em cada 100 mortes (1,3\%) em 2019 foram resultado de suicídio (WORLD HEALTH ORGANIZATION, 2019). O número de óbitos decorrentes de suicídios tem aumentado significativamente, principalmente na população mais jovem, porém considera-se que há grande possibilidade de prevenção dos casos (MACENTE LB e ZANDONADE E, 2010; SOUZA V, et al., 2011).

No Brasil, os dados sobre óbitos são compilados pelo Sistema de Informação de Mortalidade (SIM). O SIM é um sistema de vigilância epidemiológica desenvolvido com o intuito de reunir, organizar e disponibilizar dados atualizados e confiáveis sobre mortalidade. Os dados utilizados pelo SIM são coletados a partir da Declaração de Óbito (DO). Para garantir uma igualdade nos dados coletados a DO é um documento padronizado em todo o território nacional. Após compilação, os dados advindos do SIM são disponibilizados no site do DATASUS permitindo acesso às informações sobre mortalidade em nível nacional, regional, estadual e municipal. (MINISTÉRIO DA SAÚDE, 2001).

Em números absolutos o Brasil é um dos países da América Latina com maiores índices de casos de óbitos causados por suicídios (BOTEGA NJ, 2014). Dados nacionais englobando o período de 2011 a 2017, demonstram que foram registrados 80.352 óbitos por suicídio na população brasileira a partir de 10 anos (MINISTÉRIO DA SAÚDE, 2019).

A região Sul do Brasil, apesar de concentrar apenas $14 \%$ da população do país, concentra $23 \%$ dos registros de suicídio. Os dados disponibilizados pelo DATASUS demonstram que em Santa Catarina no período de 2011 a 2017, foram notificados 4.273 óbitos por suicídio havendo aumento na taxa de mortalidade de 8,3 para 10,4 óbitos a cada 100 mil habitantes no período estudado (MINISTÉRIO DA SAÚDE, 2021; SANTA CATARINA, 2019). Pode-se observar que a taxa de mortalidade por suicídio em Santa Catarina encontrava-se superior aos valores brasileiros que foram de 5,3 e 6,14 óbitos a cada 100 mil habitantes em 2011 e 2017 respectivamente (MINISTÉRIO DA SAÚDE, 2019; MINISTÉRIO DA SAÚDE, 2017).

Levando-se em conta os dados alarmantes sobre suicídio, torna-se necessária a implementação de medidas preventivas efetivas que devem ser elaboradas com base no perfil epidemiológico desses óbitos considerando a realidade local. Dessa forma, o presente estudo teve como objetivo avaliar a prevalência de suicídio na cidade de Joinville, Santa Catarina, no período de 2016 a 2018 e o perfil epidemiológico dos pacientes que cometeram suicídio no referido período.

\section{MÉTODOS}

Estudo descritivo do perfil epidemiológico dos indivíduos que foram a óbito por suicídio na cidade de Joinville, no período de 2016 a 2018. Localizado na Região Norte do Estado de Santa Catarina, o município de Joinville possui uma população estimada em 590.466 habitantes, cujas residências se localizam, em sua maioria, dentro do perímetro urbano. Grande parte das atividades relacionadas ao setor econômico, estão no setor secundário, com destaque para as indústrias de transformação além do comércio e prestação de serviços (PREFEITURA DE JOINVILLE, 2020).

A partir dos dados disponibilizados pelo no Sistema de Informação de Mortalidade (SIM) foi possível identificar o número de óbitos ocorridos em Joinville durante o período estabelecido devido ao suicídio. Esses 
óbitos foram classificados segundo a décima revisão da Classificação Estatística Internacional de Doenças e Problemas Relacionados à Saúde (CID-10). O Sistema também permitiu coleta de informações referente ao sexo, idade, raça, escolaridade, bairro de moradia, local e forma de ocorrência do suicídio. Realizou-se análise descritiva das características sociodemográficas (raça/cor, escolaridade, sexo e idade) e de ocorrência (meio utilizado e local) dos óbitos.

Para apresentação dos resultados, calculou-se a taxa de mortalidade específica por suicídio dividindo-se o número de óbitos pela população residente. Foram calculadas as taxas de mortalidade específicas segundo sexo (masculino e feminino), dividindo-se o número de óbitos segundo o sexo pela população masculina ou feminina a cada ano avaliado. As taxas de mortalidade por faixa etária (16 a 19; 20 a 29; 30 a 39; 40 a 49; 50 a 59; 60 a 69; 70 anos ou mais) foram calculadas dividindo-se o número de óbito em cada faixa etária pela população de cada faixa etária correspondente. As estimativas populacionais foram obtidas do Instituto Brasileiro de Geografia e Estatística (IBGE).

Dados sobre óbitos ocorridos no Brasil, na Região Sul e em Santa Catarina, e causados por Lesões autoprovocadas voluntariamente (CID 10 X60-X84) foram obtidos através do DATASUS. A categorização segundo a profissão de cada caso analisado foi realizada de acordo com o Código Brasileiro de Ocupações. A pesquisa foi aprovada pelo Comitê de Ética em Pesquisa da Universidade da Região de Joinville (Univille), segundo o parecer n. 4.547.509.

\section{RESULTADOS}

Durante o período estabelecido para a coleta dos dados foram identificados 140 óbitos devido à suicídios na cidade de Joinville, sendo 48 em 2016, 50 em 2017 e 42 em 2018. O perfil das pessoas que cometeram suicídio no período estudado era majoritariamente do sexo masculino (83\%), brancos (86\%), que concluíram o ensino médio (45\%), e de situação conjugal solteira, viúva ou divorciada $(69,2 \%)$ sem variações significativas ao se analisar esses dados a cada ano.

Ao se calcular a taxa de mortalidade por suicídio pode-se verificar uma redução de 8.4 óbitos a cada 100 mil habitantes em 2016 para 7,2 óbitos a cada 100 mil habitantes em 2018. Essa redução não foi observada ao se comparar as taxas de mortalidade de Joinville às taxas do Brasil, da Região Sul do país e de Santa Catarina como pode ser visualizado no Gráfico 1.

Gráfico 1 - Taxas de mortalidade por suicídio a cada 100.000 habitantes encontradas no Brasil, Região Sul, Santa Catarina e Joinville e 2016 a 2018.

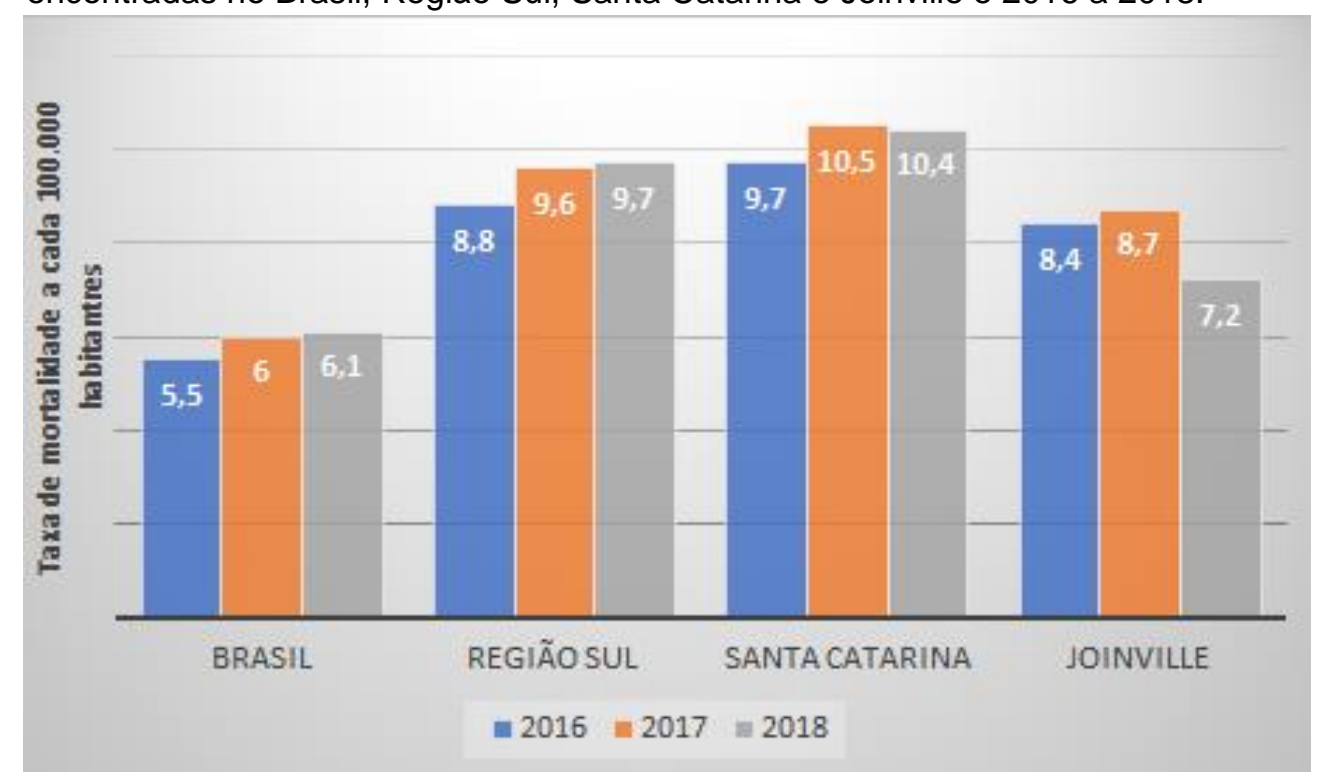

Fonte: Plucinski A, et al., 2021; dados extraídos do departamento de informática do Sistema Único de Saúde do Brasil (DATASUS) e IBGE. 
Como mencionado anteriormente, da totalidade dos 140 óbitos, $83 \%(n=116)$ ocorreram em homens e $17 \%(n=24)$ em mulheres, sendo que esses valores não se alteraram significativamente quando analisaramse os dados de cada ano em separado. A taxa de mortalidade em homens no ano de 2016 foi de 14,16 óbitos a cada 100.000 habitantes, caindo para 12,4 óbitos a cada 100.000 habitantes no ano de 2018 . No caso das mulheres houve redução de 2,8 óbitos a cada 100.000 habitantes em 2016 para 2,04 óbitos a cada 100.000 habitantes em 2018 (Tabela 1).

Tabela 1 - Número de óbitos e taxa de mortalidade a cada 100 mil habitantes segundo o sexo no período de 2016 a 2018.

\begin{tabular}{ccccc}
\hline \multirow{2}{*}{ Ano } & \multicolumn{2}{c}{ Número de óbitos } & \multicolumn{2}{c}{ Taxa de mortalidade } \\
\cline { 2 - 5 } & Homens & Mulheres & Homens & Mulheres \\
\hline 2016 & 40 & 8 & 14,1 & 2,8 \\
2017 & 40 & 10 & 13,9 & 3,5 \\
2018 & 36 & 6 & 12,4 & 2,04 \\
\hline
\end{tabular}

Fonte: Plucinski A, et al., 2021; dados extraídos do Sistema de Mortalidade (SIM) e IBGE.

$\mathrm{Na}$ análise dos óbitos por faixa etária, foi possível verificar que ainda que os números absolutos apontassem para uma maior ocorrência de casos em faixas etárias mais jovens, dos 20 aos 39 anos, as taxas específicas demonstraram maior concentração de casos na faixa dos 60 aos 69 anos em 2016 e 2017 e dos 70 aos 79 anos em 2018. Pode-se observar um aumento nas taxas de mortalidade nos extremos de idade nos anos analisados (Tabela 2).

Tabela 2 - Taxa de mortalidade a cada 100 mil habitantes calculada por faixa etária no período de 2016 a 2018.

\begin{tabular}{cccc}
\hline Faixa Etária & $\mathbf{2 0 1 6}$ & $\mathbf{2 0 1 7}$ & $\mathbf{2 0 1 8}$ \\
\hline $16-19$ anos & 9,08 & 11,7 & 14,5 \\
$20-29$ anos & 6,1 & 11,1 & 11,1 \\
$30-39$ anos & 13,2 & 5,0 & 6,9 \\
$40-49$ anos & 8,6 & 12,1 & 4,8 \\
$50-59$ anos & 12,1 & 13,2 & 11,5 \\
$60-69$ anos & 19,8 & 16,4 & 6,7 \\
$70-79$ anos & 5,7 & 16,0 & 15,0 \\
\hline
\end{tabular}

Fonte: Plucinski A, et al., 2021; dados extraídos do Sistema de Mortalidade (SIM) e IBGE.

Ao se analisarem os dados por sexo e faixa etária em conjunto verificou-se que nos anos de 2016 e 2017 , as maiores taxas de mortalidade foram encontradas nos homens na faixa dos 60-69 anos, 37.5 e 30.5 óbitos a cada 100 mil habitantes respectivamente. Em 2018, homens com idade de 16 a 19 anos foram os mais afetados, seguidos por aqueles com idade de 70 a 79 anos. Ainda que as mulheres tenham apresentado taxas mais baixas, pode-se observar uma maior frequência dos casos na faixa dos 50-59 anos em 2016 dos 70-79 anos em 2017 e 2018 (Tabela 3).

Tabela 3 - Taxas de mortalidade a cada 100 mil habitantes de acordo com o sexo e faixa etária.

\begin{tabular}{ccccccc}
\hline \multirow{2}{*}{ Faixa Etária } & \multicolumn{3}{c}{ Homens } & \multicolumn{3}{c}{ Mulheres } \\
\cline { 2 - 7 } & $\mathbf{2 0 1 6}$ & $\mathbf{2 0 1 7}$ & $\mathbf{2 0 1 8}$ & $\mathbf{2 0 1 6}$ & $\mathbf{2 0 1 7}$ & $\mathbf{2 0 1 8}$ \\
\hline $16-19$ & 13,3 & 18,3 & $\mathbf{2 8 , 3}$ & 4,6 & 4,8 & 0 \\
$20-29$ & 11,8 & 17,8 & 19,8 & 0 & 4,1 & 2,1 \\
$30-39$ & 22,1 & 5,9 & 13,6 & 4,1 & 4,1 & 0 \\
$40-49$ & 14,9 & 22,1 & 4,84 & 2,4 & 2,4 & 4,7 \\
$50-59$ & 15,8 & 21,5 & 18,02 & 8,7 & 5,6 & 5,5 \\
$60-69$ & 37,5 & 30,5 & 14,5 & 4,6 & 4,4 & 0 \\
\hline
\end{tabular}

Fonte: Plucinski A, et al., 2021; dados extraídos do Sistema de Mortalidade (SIM) e IBGE. 
Outro ponto analisado foi a área do município em que ocorreu o óbito. Não houve uma região que se destacou por apresentar taxas de mortalidade mais altas que as demais em todos os anos analisados, observando-se uma variação da região do município com maior taxa a cada ano. Nas regiões oeste e sudeste houve redução das taxas de mortalidade a cada ano. Uma redução de taxas de 2016 para 2017 com tendência a estabilização de 2017 para 2018 foi verificada na região leste. Já na região centro-norte, após um aumento da taxa de 2016 para 2017 houve estabilização dos valores de 2017 para 2018 . Houve redução nos valores de 2016 para 2017 e aumento de 2017 para 2018 nas regiões nordeste, sudoeste e de Pirabeiraba. Por fim, a região sul apresentou aumento de 2016 para 2017 com redução no ano subsequente (Tabela 4).

Tabela 4 - Taxa de mortalidade por 100 mil habitantes de acordo com a região do município de Joinville no período de 2016 a 2018.

\begin{tabular}{cccc}
\hline Região do município & $\mathbf{2 0 1 6}$ & $\mathbf{2 0 1 7}$ & $\mathbf{2 0 1 8}$ \\
\hline Centro-Norte & 3,65 & 10,7 & 10 \\
Leste & 10 & 8,8 & 8,8 \\
Nordeste & 11,1 & 6,3 & 11 \\
Oeste & 12,3 & 12,2 & 4 \\
Pirabeiraba & 26,1 & 0 & 8,6 \\
Sudeste & 14,2 & 11,4 & 6,3 \\
Sudoeste & 12 & 7,9 & 19,7 \\
Sul & 8,7 & 11,8 & 6,4 \\
\hline
\end{tabular}

Fonte: Plucinski A, et al., 2021; dados extraídos do Sistema de Mortalidade (SIM) e IBGE.

A análise do meio utilizado para se cometer o suicídio, demonstrou predominância do CID X70 - Lesão autoprovocada intencionalmente por enforcamento, estrangulamento e sufocação, responsável por 91 (65\%) dos 140 casos analisados.

Com relação aos locais de ocorrência dos suicídios pode-se constatar que a maioria ocorreu na residência (62\%), porém houve redução desse percentual ao longo dos anos de 79,2\% em 2016 para $43 \%$ em 2018. Em 2018, 31\% dos casos apresentavam CID referente a outros locais especificados e local não especificado.

Sobre a ocorrência dos óbitos, em 2016, $89,5 \%$ ocorreram no domicílio e os demais em hospitais. Em 2017, óbitos em domicílio somaram 56\%, foram contabilizados óbitos em outros estabelecimentos (24\%), ocorrência de óbitos em hospitais foi responsável por $16 \%$ dos casos e os demais ocorreram em vias públicas. Em 2018, 64\% dos óbitos ocorreram no domicílio, 21\% em outros estabelecimentos, 9,5\% em hospitais e os demais ocorreram em vias públicas.

As ocupações mais encontradas foram estudante $(n=12)$, operador de máquinas fixas em geral $(n=6)$, alimentador de linha de produção $(n=6)$, dona de casa $(n=6)$, representante comercial autônomo $(n=6)$, vigilante $(n=5)$, mecânico de automóveis, motocicletas e veículos similares $(n=4)$, motorista de furgão ou veículo similar $(n=4)$, pedreiro $(n=4)$, enfermeiro $(n=3)$ e pintor de obras $(n=3)$.

\section{DISCUSSÃO}

Aproximadamente 800.000 pessoas cometem suicídio a cada ano sendo a redução das taxas de mortalidade por essa causa uma meta global priorizada pela OMS e incluída como um indicador nos Objetivos de Desenvolvimento Sustentável das Nações Unidas (indicador 3.4.2.) (WORLD HEALTH ORGANIZATION, 2014; INSTITUTO DE PESQUISA ECONÔMICA APLICADA, 2018).

No presente estudo foi verificado uma redução na taxa de mortalidade por suicídio na cidade de Joiniville de 2016 a 2018. A queda na taxa pode ser resultado do trabalho preventivo desenvolvido pelo Núcleo de Prevenção de Violência (NPV). Quando um paciente dá entrada em algum dos hospitais da cidade por tentativa de suicídio, o médico é orientado a fazer a ficha de notificação que é encaminhada para o NPV e para o Centro de Atenção Psicossocial que fará o seguimento do paciente visando evitar novas tentativas 
(PREFEITURA DE JOINVILLE, 2020). Ainda que tenha ocorrido queda, as taxas encontradas na Região Sul e em Joinville são superiores à média nacional demonstrando a necessidade de se analisar o perfil das pessoas que cometem suicídio e compreender as causas que norteiam esses atos visando reduzir as taxas através de políticas voltadas a prevenção (MINISTÉRIO DA SAÚDE, 2021; SANTA CATARINA, 2019).

Dados da análise das taxas de suicídio específicas por faixa etária em Joinville demonstraram maior prevalência entre 60 aos 69 anos nos anos de 2016 e 2017 e na faixa dos 70 a 79 anos em 2018. Segundo o Ministério da Saúde, os números de 2018 apontam para a alta taxa de suicídio entre aqueles com mais de 70 anos sendo registrada a taxa média de 8,9 mortes por 100 mil nos últimos seis anos estando acima da taxa média nacional que é 5,5 por 100 mil (MINISTÉRIO DA SAÚDE, 2020). Dados semelhantes foram encontrados no estudo realizado por Benetti IC, et al.(2018) que concluiu que as taxas de óbito por suicídio em Santa Catarina aumentaram conforme a idade durante o período analisado.

Segundo o Boletim Epidemiológico da Diretoria de Vigilância Epidemiológica de Santa Catarina, houve aumento nas taxas de mortalidade por suicídio em homens conforme aumento da faixa etário no período de 2011 a 2017. Benetti IC, et al (2018) também afirmam que em seu estudo a taxa de mortalidade foi três a quatro vezes maior no sexo masculino e que no caso dos homens, as taxas foram ascendentes atingindo os maiores valores nas faixas acima de 80 anos. No presente estudo foram encontradas taxas superiores em homens em todas as faixas etárias e nos três anos analisados, mesmo padrão identificado em outros estudos como Hedegaard H, et al. (2020), Ministério da Saúde (2019) e Santa Catarina (2019). Não foi identificado um padrão ascendente, mas sim uma maior concentração de casos nos extremos de idades, vide análise de dados do presente estudo.

Segundo Cecilia L (2021), não existe uma única explicação para os homens cometerem um número maior de suicídios, mas sim um contexto social de excessivas cobranças, sofrimentos, o "papel de provedor da família" que os levam ao abuso de substâncias primariamente e futuramente a autolesão. Homens possuem maior dificuldade em falar sobre si e sobre seus sentimentos, podem reagir de forma hostil e competitiva frente a situações difíceis e são mais resistentes à ideia de uma ajuda profissional (NUNES AM, 2018; MACHADO DB e SANTOS DN, 2015). O maior número de óbitos em homens também pode ser explicado por argumentos biologicistas que incluem a maior letalidade dos meios usados por eles ao cometerem suicídio (CALLANAN VJ e DAVIS MS, 2012).

A literatura aponta que grande parte dos homens idosos que cometeram suicídio, foram instigados por questões sociais e/ou médicas envolvidas no contexto do episódio, como: perdas de bens, status, honra e prejuízos nos empreendimentos e/ou enfermidades que os acometeram (MINAYO MC, et al., 2012). Nas mulheres as faixas mais encontradas foram 50-59 em 2016 e 70-79 nos outros dois anos analisados em Joinville. O suicídio em mulheres idosas ocorre quando elas não conseguem mais cumprir o seu papel de cuidadora (MENEGHEL SN, et al., 2012).

Entretanto, analisando de um extremo de idade a outro, a faixa etária e ocupação merecedoras de grande atenção devido suas taxas de óbitos em crescente, são os jovens e estudantes. Globalmente, os suicídios são a segunda principal causa de mortalidade prematura em indivíduos de 15 a 29 anos (precedidos por acidentes de trânsito), e o número três na faixa etária de 15 a 44 anos (BERTOLOTE JM e FLEISCHMANN A, 2002).

Diversas mudanças na vida de jovens e adultos emergentes podem contribuir e dificultar a vida, a ponto de o suicídio ser considerado a única alternativa. Fatores de proteção como autoestima, autoeficácia, habilidades sociais, relacionamentos familiares e amizades, são importantes e essenciais para que o indivíduo não apresente ideação suicida. Importante também para que após uma tentativa fracassada, não aconteça novamente (PEREIRA AS, et al. 2021).

Com relação ao meio utilizado para cometer o suicídioem Joinville, encontrados predominância do CID X70 - Lesão autoprovocada intencionalmente por enforcamento, estrangulamento e sufocação, responsável por 91 (65\%) dos 140 casos analisados. Segundo estudo realizado pelo Ministério da Saúde sobre o Perfil epidemiológico dos casos notificados de violência autoprovocada e óbitos por suicídio entre 2011 a 2018, 
houve predomínio de enforcamento, seguido de intoxicação exógena e uso de armas de fogo. Uma revisão conduzida em ao avaliar os métodos utilizados para se cometer suicídio detectou variações dependendo da região em que ocorriam. Os autores observaram predomínio de óbitos por armas de fogo nos Estados Unidos, enforcamentos, envenenamentos e quedas em países asiáticos. O enforcamento também predominou em vários países europeus (BACHMANN S, 2018).

Quando analisadosos dados de Joinville, não há uma região da cidade que se sobressaia apresentando taxas mais constantemente mais elevadas que as demais e necessitando de uma maior atenção. Entretanto, o perfil epidemiológico de homens, em extremos de idades, como discutido durante a análise de dados desse artigo, deve sim ser o ponto de atenção do âmbito responsável.

A partir do momento que observamos um perfil populacional, a ideia é encontrar maneiras para impedir que esses atos ocorram. A atenção primária, sendo a porta de entrada para essa população deve estar preparada para acolher e entender o gatilho inserido no ambiente desses pacientes, para serem implantadas e praticadas medidas que previnam os atos. A intenção deve ser ampliar acesso à atenção psicossocial, ações intersetoriais, capacitações dos profissionais, atendimento multiprofissional e organizar demandas de assistência (FERREIRA GS, 2019).

\section{CONCLUSÃO}

A análise dos dados realizada pelo presente trabalho auxilia na compreensão do fenômeno, pois ao se descrever o perfil epidemiológico é possível a elaboração de estratégias de prevenção de acordo com as características locais. Na cidade de Joinville o atendimento pós-tentativa de suicídio pode ter contribuído com a redução da taxa de mortalidade de 2016 a 2018, porém a ascensão da taxa de mortalidade por suicídio em jovens e idosos deixa clara a importância da contínua construção de uma rede para prevenção e acolhimento ao paciente com ideação suicida.

\section{REFERÊNCIAS}

1. BACHMANN S. Epidemiology of Suicide and the Psychiatric Perspective. Int J Environ Res Public Health, 2018;15(7): 1425

2. BENETTI IC, et al. Características do suicídio em Santa Catarina: um estudo do período de 2007 a 2016. Estud. Psicol., 2018; 23 (4): 404-415.

3. BERTOLOTE JM, FLEISCHMANN A. A global perspective in the epidemiology of suicide. Suicidology. 2002; 7:6-8

4. BOTEGA NJ. Comportamento suicida: epidemiologia. Psicologia USP [online], 2014; 25(3): 231-236.

5. CALLANAN VJ, DAVIS MS. Gender differences in suicide methods. Soc Psychiatry Psychiatr Epidemiol, 2012; 47(6): 857-69

6. CECILIA, L. Tentativa de suicídio: um estudo das publicações brasileiras. Brazilian journal of health review, 2021; 4: 3425-3433.

7. DAOLIO ER, SILVA, JV. Os significados e os motivos do suicídio: As representações sociais de pessoas residentes em Bragança Paulista, SP. Bioethikos, 2009; 3(1): 68-76.

8. FERREIRA GS, et al. Possibilidades de abordagem do tema do suicídio na Estratégia Saúde da Família. Physis: Revista de Saúde Coletiva [online], 2019; 29(4).

9. HEDEGAARD, HOLLY, et al. Increase in Suicide Mortality in the United States, 1999-2018. NCHS, $2020 ; 362$

10. INSTITUTO DE PESQUISA ECONÔMICA APLICADA. Agenda 2030. ODS - Metas Nacionais dos Objetivos de Desenvolvimento Sustentável Proposta de adequação. Brasília: Ministério do Planejamento, Desenvolvimento e Gestão, $\quad 2018 ; \quad 505 \quad$ p. $\quad$ Disponível http://repositorio.ipea.gov.br/bitstream/11058/8636/1/Agenda\%202030\%200DS\%20Metas\%20Nac\%20dos\%20Obj \%20de\%20Desenv\%20Susten\%202018.pdf. Acesso em: 20 mai 2021.

11. MACENTE LB, ZANDONADE E. Avaliação da completude do sistema de informação sobre mortalidade por suicídio na região Sudeste, Brasil, no período de 1996 a 2007. Jornal Brasileiro de Psiquiatria, 2010; 59(3),173-181.

12. MACHADO DB, SANTOS DN. Suicídio no Brasil, de 2000 a 2012 . J Bras Psiquiatr. 2015; 64(1):45-54.

13. MINISTÉRIO DA SAÚDE. Secretaria de Vigilância em Saúde. Perfil epidemiológico dos casos notificados de violência autoprovocada e óbitos por suicídio entre jovens de 15 a 29 anos no Brasil, 2011 a 2018. Boletim Epidemiológico. 2019. Disponível em: https://antigo.saude.gov.br/images/pdf/2019/setembro/13/BE-suic--dio-24-final.pdf. Acesso em: 20 mai 2021.

14. MINISTÉRIO DA SAÚDE. Manual de procedimento do sistema de informações sobre mortalidade: Brasília: Ministério da Saúde: Fundação Nacional de Saúde, 2001; 36 p. Disponível em: https://bvsms.saude.gov.br/bvs/publicacoes/sis_mortalidade.pdf. Acesso em: 20 mai 2021. 
15. MINISTÉRIO DA SAÚDE. Banco de dados do Sistema Único de Saúde - DATASUS, Informações em Saúde, Sistema de Informações sobre Mortalidade. Disponível em: https://datasus.saude.gov.br/mortalidade-desde-1996-pela-cid-10. Acesso em: 21 jul 2021.

16. MINISTÉRIO DA SAÚDE. Secretaria de Vigilância em Saúde. Suicídio. Saber, agir, prevenir. Boletim Epidemiológico. Brasília: Ministério da Saúde, 2017; 15 p. Disponível em: https://portalarquivos2.saude.gov.br/images/pdf/2017/setembro/21/2017-025-Perfil-epidemiologico-das-tentativas-eobitos-por-suicidio-no-Brasil-e-a-rede-de-atencao-a-saude.pdf. Acesso em 21 jul 2021.

17. MINISTÉRIO DA SAÚDE. Suicídio na Pandemia. Saúde Mental e Atenção Psicossocial na Pandemia Covid-19. Fundação Oswaldo Cruz, 2020; 24p Disponível em: https://www.fiocruzbrasilia.fiocruz.br/wpcontent/uploads/2020/04/Sa\%C3\%BAde-Mental-e-Aten\%C3\%A7\%C3\%A3o-Psicossocial-na-Pandemia-Covid-19recomenda\%C3\%A7\%C3\%B5es-gerais.pdf. Acesso em: 20 jul 2021.

18. MENEGHEL SN et al. Suicídio de idosos sob a perspectiva de gênero. Ciência \& Saúde Coletiva [online], 2012; 17(8): 1983-1992.

19. MINAYO MCS, et al. Suicídio de homens idosos no Brasil Suicide of elderly men in Brazil. Ciência \& Saúde Coletiva, 2012; 17(10): 2665-2674.

20. NUNES AM. Suicide in Portugal: image of the country. J Bras Psiquiatr. 2018; 67(1):25-33.

21. PEREIRA AS, et al. Risk and protective factors for suicide attempt in emerging adulthood. Ciênc. saúde coletiva, 2018; 23(11): 3767-3777..

22. PREFEITURA DE JOINVILLE. Secretaria de Planejamento Urbano e Desenvolvimento Sustentável. Joinville Cidade em Dados. 2020. Disponível em https://www.joinville.sc.gov.br/publicacoes/joinville-cidade-em-dados-2020/. Acesso em 21 Jul 2021.

23. RIBEIRO NM, et al. Análise da tendência temporal do suicídio e de sistemas de informações em saúde em relação às tentativas de suicídio. Texto contexto - enfermagem, 2018; 27(2): 2110016.

24. SANTA CATARINA. Barriga Verde Informativo Epidemiológico. Perfil epidemiológico das tentativas e de óbitos por suicídio no estado de Santa Catarina e a rede de atenção à saúde (2012-2017), 2019. Disponível em: http://www.dive.sc.gov.br/barrigaverde/pdf/BarrigaVerde\%20Suicidio.pdf. Acesso em: 12 jan 2021.

25. SCHLOSSER A, et al. Revisão: comportamento suicida ao longo do ciclo vital. Temas psicol., 2014; 22(1):133-145.

26. SOUZA VS. et al. Tentativas de suicídio e mortalidade por suicídio em um município no interior da Bahia. Jornal Brasileiro de Psiquiatria, 60(4),294-300.

27. WORLD HEALTH ORGANIZATION. Suicide worldwide in 2019: global health estimates. Geneva: World Health Organization, 2021. Disponível em: https://www.who.int/teams/mental-health-and-substance-use/suicide-data. Acesso em: 21 jul 2021.

28. WORLD HEALTH ORGANIZATION. Preventing suicide: a global imperative. World Health Organization. Geneva: World Health Organization, 2014. Disponível em: https://apps.who.int/iris/handle/10665/131056. Acesso em: 10 abril 2021. 